${ }^{1}$ D. V. Lang, J. Appl. Phys. $\underline{45}, 3014$ (1974), and $\underline{47}$, 3587 (1976).

${ }^{2}$ D. Pons and S. Makram-Ebeid, J. Phys. (Paris) 40 , 1161 (1979) .

${ }^{3}$ S. Makram-Ebeid, Appl. Phys. Lett. 37, 464 (1980), and in Defects in Semiconductors, edited by J. Narayan and T.Y. Tan (North-Holland, New York, 1981), Vol. 2, p. 495 .

${ }^{4}$ S. Makram-Ebeid, G. M. Martin, and D. W. Woodard, J. Phys. Soc. Jpn. $\underline{49}, 287$ (1980) .

${ }^{5} \mathrm{~J}$. R. Oppenheimer, Phys. Rev. 31, 66 (1928).

${ }^{6} \mathrm{~K}$. Huang and A. Rhys, Proc. Roy. Soc. London, Ser. A 204,406 (1950).

${ }^{7} \mathrm{~S}$. Makram-Ebeid and M. Lannoo, to be published.

${ }^{8}$ A. M. Stoneham, Theory of Defects in Solids (Clarendon, Oxford, 1975).

${ }^{9}$ M. G. Burt, J. Phys. C $\underline{12}, 4827$ (1979).
${ }^{10}$ C. H. Henry and D. V. Lang, Phys. Rev. B 15, 989 (1977) .

${ }^{11} \mathrm{D}$. Bois and A. Chantre, Rev. Phys. Appl. 15, 631 (1980).

${ }^{12}$ T. Jesper, B. Hamilton, and A. R. Peaker, in SemiInsulating III-V Materials, edited by G. J. Rees (Shiva Publishing LTD, Orpington, United Kingdom, 1980), p. 233 .

${ }^{13}$ P. Leyral, F. Litty, S. Loualiche, A. Nouailhat, and G. Guillot, to be published.

${ }^{14}$ V. Piekara, J. M. Langer, and E. B. KrulowskaFulde, Solid State Commun. 23, 583 (1977).

${ }^{15}$ A. M. Hennel, W. Szuskiewicz, G. Martinez, and B. Clerjaud, Rev. Phys. Appl. 15, 697 (1980).

${ }^{16}$ B. K. Ridley, J. Phys. C 11,2323 (1978).

${ }^{17}$ A. Mitonneau, A. Mircea, G. M. Martin, and D. Pons, Rev. Phys. Appl. 14, 853 (1979).

\title{
Stress Tuning of the Metal-Insulator Transition at Millikelvin Temperatures
}

\author{
M. A. Paalanen, (a) T. F. Rosenbaum, G. A. Thomas, and R. N. Bhatt \\ Bell Laboratories, Murray Hill, New Jersey 07974 \\ (Received 26 February 1982)
}

\begin{abstract}
A high-resolution scan of the metal-insulator transition in Si:P at millikelvin temperatures has been obtained by applying uniaxial stress. A sharp, but continuous, metalinsulator transition is resolved, with conductivities below Mott's "minimum" value $\sigma_{\mathrm{M}}$. These measurements join smoothly with previous low-resolution experiments, ruling out any discontinuity at $\sigma_{\mathrm{M}}$. The reproducible critical behavior dis agrees with predictions of existing scaling theories of localization.
\end{abstract}

PACS numbers: $71.30 .+\mathrm{h}, 72.15 . \mathrm{Cz}, 72.20 . \mathrm{Fr}$

Our understanding of the metal-insulator transition in random systems rests in part on Anderson's demonstration ${ }^{1}$ of the absence of electronic diffusion at zero temperature in a sufficiently strong random potential and in part on the work of $\mathrm{Mott}^{2}$ and Hubbard ${ }^{3}$ emphasizing the role of electron correlations in stabilizing the insulating state. This transition has been observed in different materials at electron concentrations $n_{c}$ varying over eight orders of magnitude, ${ }^{4}$ but with a universal scaling for which Mott has argued using simple models:

$$
n_{c}{ }^{1 / 3} a_{\mathrm{B}} \simeq \frac{1}{4},
$$

where $a_{\mathrm{B}}$ is the radius of the isolated impurity wave function. A prototype of such systems is a random array of $\mathbf{P}$ donors placed substitutionally into a crystalline Si lattice.

Following these ideas Mott suggested ${ }^{5}$ in 1972 that the zero-temperature conductivity $\sigma(0)$ jumped from zero in the insulator to a minimum metallic value

$$
\sigma_{\mathrm{M}}=C_{\mathrm{M}} e^{2} / \hbar n_{c}^{-1 / 3},
$$

where $C_{M}=\frac{1}{20}$ within a factor of 2. For Si:P, $\sigma_{M}$ $=20(\Omega \mathrm{cm})^{-1}$. According to Mott's recent survey, ${ }^{6}$ all existing data support this remarkable conclusion.

In contrast, the single-particle scaling theories of localization ${ }^{7}$ (neglecting electron-electron interactions) predict a continuous variation through the critical region, i.e., $\sigma(0)<\sigma_{M}$, of the form

$$
\sigma(0) \simeq \sigma_{M}\left(n / n_{c}-1\right)^{\nu},
$$

where $\nu \approx 1$. Although experimental studies ${ }^{8,9}$ of $\mathrm{Si}: \mathrm{P}$ and $\alpha-\mathrm{Ge}_{1-x} \mathrm{Au}_{x}$ have found $\sigma(0)$ values significantly less than $\sigma_{M}$, Mott has argued ${ }^{6}$ correctly that macroscopic inhomogeneities could have played a dominant role for $\sigma(0)<\sigma_{M}$. Furthermore, in Si:P, the sharpness of the transition $^{8}$ precluded resolution of this critical region. Thus, Eqs. (2) and (3) have escaped a definitive 
test.

We report here a high-resolution, zero-temperature study of the metal-insulator transition, using uniaxial compressive stress $S$ to tune $n_{c}$ through $n$ of uncompensated, slightly insulating $\mathrm{Si}: \mathrm{P}$ samples. We find reproducible behavior for $n / n_{c}-1>10^{-3}$ of the form $\sigma(0) \propto\left(S-S_{c}\right)^{\nu}$, with $\nu=0.48 \pm 0.07$, which is distinguishable from apparent rounding of the transition at lower $n / n_{c}$. The reproducible behavior is consistent with previous findings ${ }^{8}$ for $\sigma(0)>\sigma_{M}$ which gave $\sigma(0)$

$=13 \sigma_{M}\left(n / n_{c}-1\right)^{\nu}$, with $\nu=0.55 \pm 0.10$. We claim that the reproducibility and consistency identify intrinsic behavior; the data thus rule out Mott's minimum metallic conductivity and are inconsistent with existing scaling theories of localization.

The capability of significantly varying $n_{c}$ with modest stresses ${ }^{10}$ relies both on the small ener-

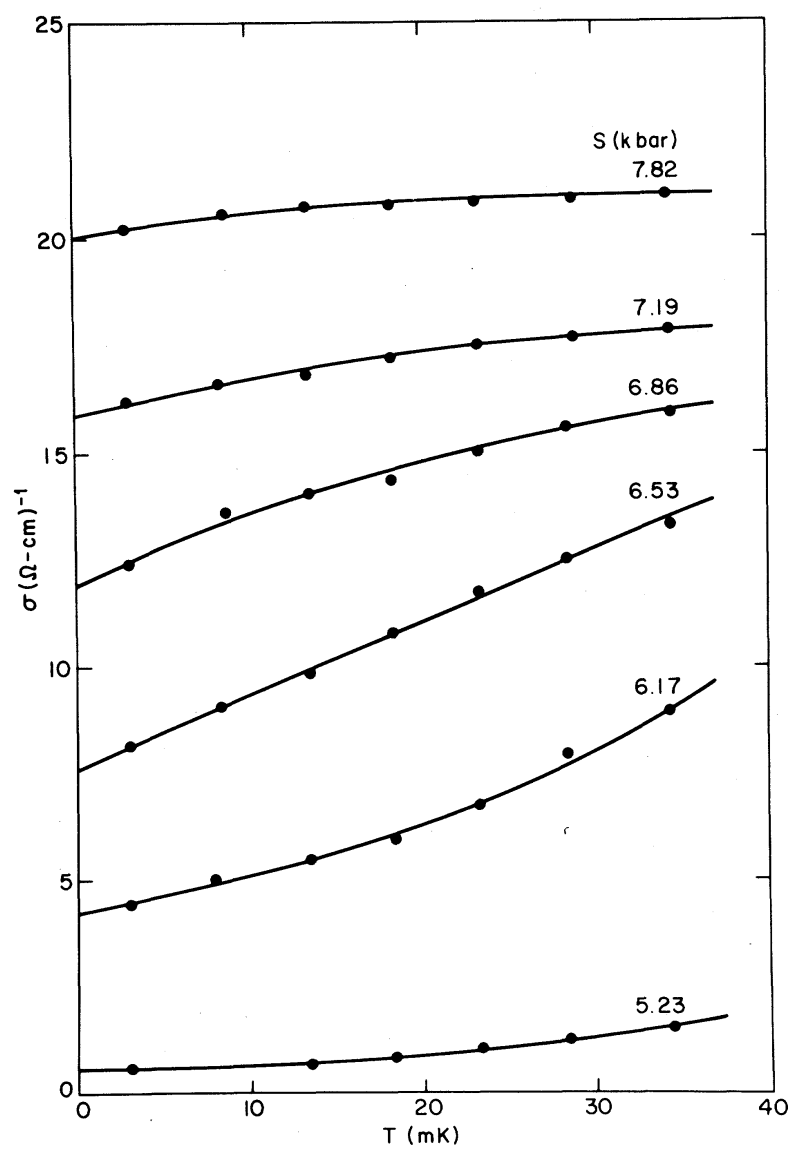

FIG. 1. Conductivity $\sigma$ of a sample of Si:P as a function of temperature $T$ for a series of values of uniaxial stress $S$ near the metal-insulator transition. The solid lines are fits by the form $\sigma(T)=\sigma(0)+B T^{\beta}$ where $\sigma(0)$, $B$, and $\beta$ are fitting parameters. gy scale of the donor band $(\sim 1 \mathrm{meV}$, rather than typical electronic energies $\sim 1 \mathrm{eV}$ ) and on the multivalley nature of the Si conduction band. The latter allows a direct coupling of the donor wave function $^{10}$ and thus the width of the donor band to uniaxial deformations. For effective-mass donors with a degenerate ground state, the variation of $n_{c}$ can be reduced ${ }^{11}$ to a Mott criterion [Eq. (1)] with different effective Bohr radii in the highstress (single valley) and stress-free (many valley) cases.

In $\mathrm{Si}: \mathrm{P}$ the valley degeneracy of the donor wave function is lifted at zero stress as a result of the short-range central-cell potential, which also causes a shrinkage of the ground-state wavefunction radius. Application of stress mixes in the relatively more extended excited states and reduces $n_{c}$ as per Eq. (1): An insulator is thus transformed into a metal at $T=0 \mathrm{~K}$. [This qualitative conclusion of Eq. (1) is supported by a detailed calculation ${ }^{12}$ of the effect of stress on the donor bands.] We approximate the (in general,

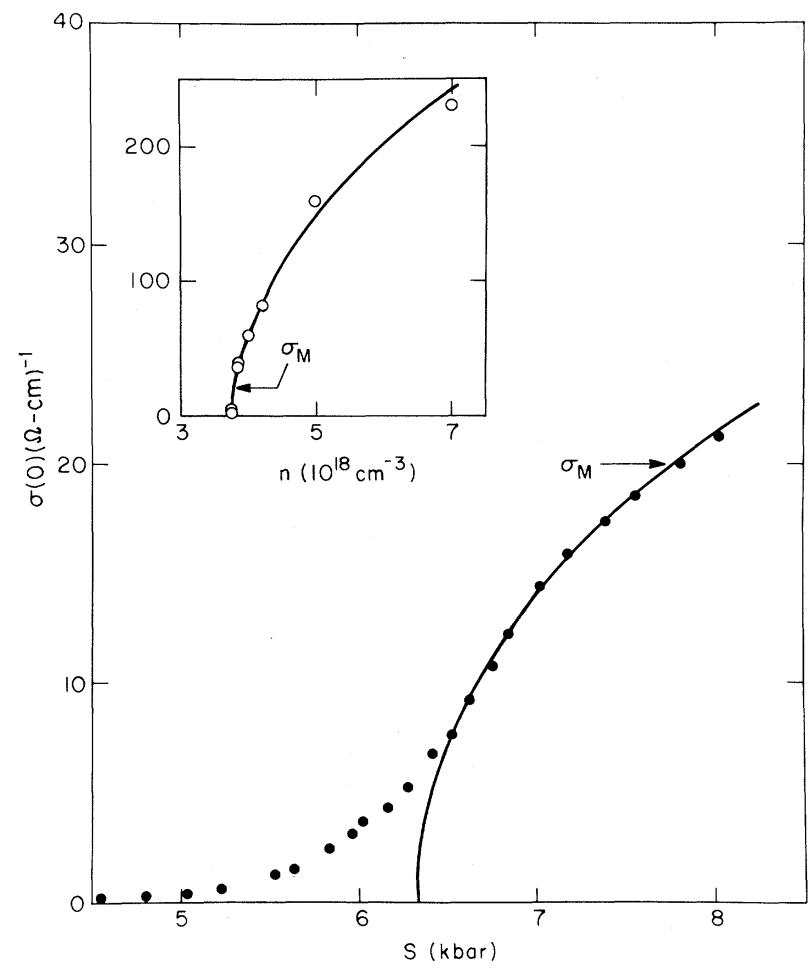

FIG. 2. Extrapolated values of zero-temperature conductivity $\sigma(0)$, obtained as illustrated in Fig. 1, as a function of uniaxial stress $S$. The solid line is fit to the region of $\sigma(0)$ which reproduces in three samples and has the form $\sigma(0) \propto\left(S-S_{c}\right)^{\nu}$ with $\nu=0.49$. The inset shows $\sigma(0)$ vs $n$ from Ref. 8 with the curve $\sigma(0)$ $=13 \sigma_{\mathrm{M}}\left(n / n_{c}-1\right)^{\nu}$ with $\nu=0.55$ 。 
nonlinear) variation of $n_{c}$ with stress $S$ by a linear relation over the critical region $(7.3 \pm 0.8 \mathrm{kbar}$ for our three samples), since it is a narrow range around a moderate value of $S$. At $4.2 \mathrm{~K}$, our measurements of conductivity in fact show an essentially linear dependence on $S$ between 4 and 12 kbar.

Our Si:P samples, with dimensions $0.3 \times 0.8$ $\times 7.0 \mathrm{~mm}^{3}$, were prepared as in Ref. 8. Two were parallel cuts from the same wafer (perpendicular to the [111] axis), and in one of the slices two sections (samples 2 and 3 ) were measured with the same current leads. For sample 3 , the voltage leads were not collinear with the current flow. The samples were mounted in a pressure device operated by ${ }^{4} \mathrm{He}$ as illustrated in the inset

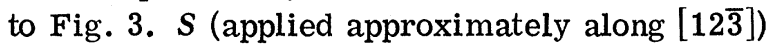
was measured capacitively at the upper end of the sample. This device was thermally anchored to a $\mathrm{Cu}$-nuclear cooling system, with $T$ measured by using a ${ }^{3} \mathrm{He}$ melting curve thermometer. The conductivity measurements were done at $11 \mathrm{~Hz}$ frequency and at power levels below $10^{-15} \mathrm{~W}$.

Sample 1 was cooled twice from room temperature with quantitatively the same results. Samples 2 and 3 also showed the same critical behavior, and only differed from each other by $15 \%$ at low $S$. This reproducibility rules out large inhomogeneities in current and $S$. After initial stress cycling, the samples showed no hysteresis in $S$ at constant $T$ to within the accuracy of our measurements $[0.5 \%$ in $\sigma(T)]$.

The variation of $\sigma(T)$ was similar for all our samples. In Fig. 1 we have plotted $\sigma(T)$ for sample 1 at values of $S$ close to the transition. The extrapolations shown (based on least-squares fits by the form in the caption) indicate metallic, i.e., finite $\sigma(0)$ values below $\sigma_{\mathrm{M}}$. Above $5(\Omega$ $\mathrm{cm})^{-1}$ the form of $\sigma(T)$ varies with $S$, but below all the samples had $\sigma(T)=\sigma(0)+A T^{2}$, as found in Ref. 8 for $\sigma(0) \lesssim \sigma_{M} / 10$. The $T^{2}$ term is not understood but a similar contribution has been shown to be related to surface conditions in insulating samples. ${ }^{13}$ For small $S$, the variation of $\sigma(0)$ (although sample dependent) is roughly exponential in $S$, inconsistent with the classical percolation ${ }^{14}$ form, $\left(n-n_{c}\right)^{1.6}$.

Figure 2 shows $\sigma(0)$ as a function of $S$ for sample 1 , while the inset exhibits the corresponding variation with $n$. By fitting the data in Fig. 2 above $6.5 \mathrm{kbar}$ and the (similar) $3-\mathrm{mK}$ data for samples 2 and 3 with the form $\left(S-S_{c}\right)^{\nu}$, we get $S_{c}(\mathrm{kbar})=6.3,6.5$, and 6.5, all \pm 0.2 , and $\nu=0.49$, 0.41 , and 0.51 , all \pm 0.07 . The quoted errors in-

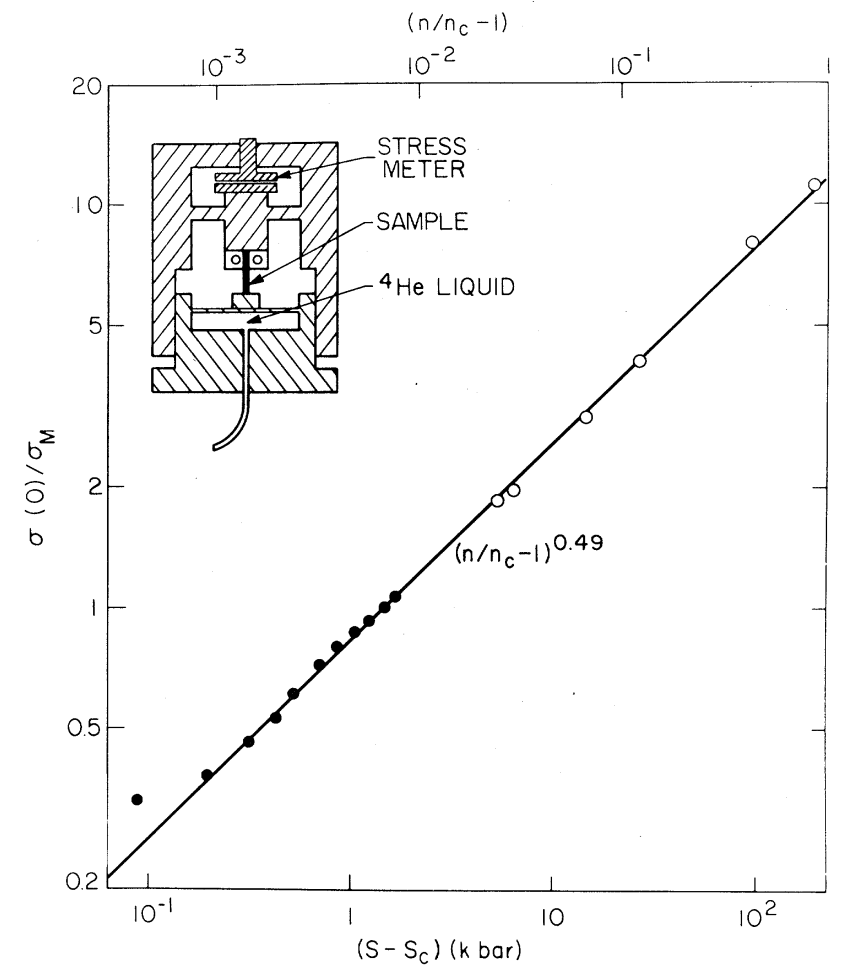

FIG. 3. A combination of the data from Fig. 2 and its inset to illustrate the smooth variations of $\sigma(0)$ through $\sigma_{\mathrm{M}}$. Values of $n / n_{c}-1$ are shown on the upper scale based on the open circles from Ref. 8. Values of $S-S_{c}$ are shown on the lower scale based on the solid circles from this work. A combination of all the results gives $\nu=0.48 \pm 0.07$.

clude not only small statistical errors, but also systematic errors inherent in the $T=0 \mathrm{~K}$ extrapolations and in the choice of the lowest $S$ value to include in the fit. We argue that the agreement among samples indicates universal behavior above $S_{c}$ in the region fitted by the solid line in Fig. 2.

If one assumes that $n_{c}$ varies linearly with $S$, our values of $\nu$ agree with the previous $\nu=0.55$ \pm 0.10 . To emphasize this agreement, we combine both sets of Fig. 2 on a log-log scale in Fig. 3 . The scaling of stress and density shown im$\operatorname{plies}^{15}\left(n / n_{c}-1\right) /\left(S-S_{c}\right)=5.4 \times 10^{-3} \mathrm{kbar}^{-1}$. The smooth variation rules out a significant change in the behavior of $\sigma(0)$ as it passes through $\sigma_{\mathrm{M}}$. The solid line is a fit by the scaling form of Eq. (3) for $\frac{1}{4} \sigma_{M} \lesssim \sigma(0) \lesssim 13 \sigma_{M}$ and $10^{-3} \lesssim n / n_{c}-1 \lesssim 1$. The wide range of this fit suggests that the characteristic conductivity of the transition region may be significantly larger than $\sigma_{M}$. This conclusion is supported by the fact that the measured conductivity deviates markedly from either that calcu- 
lated $^{8}$ for free electrons or that deduced ${ }^{6}$ from the specific heat ${ }^{16}$ below a conductivity $\sim 10 \sigma_{\mathrm{M}}$, close to the Ioffe-Regal value ${ }^{17} \sigma_{\mathrm{IR}} \simeq e^{2 / 3 \hbar n_{c}}{ }^{-1 / 3}$.

Even for $\sigma(0)<\sigma_{M}$, estimated as the critical region by current scaling theories of localization, ${ }^{7,18}$ our results give a $\nu=0.48 \pm 0.07$, inconsistent with the theoretical $\nu \approx 1$. We speculate that the more rapid variation of $\sigma(0)$ with $n-n_{c}$ arises from Coulomb effects whose importance has been emphasized by Mott. ${ }^{2,5}$ Additional evidence, attributed to Coulomb interactions in bulk systems, has come from the temperature, ${ }^{19,20}$ magnetic field ${ }^{20}$ and compensation ${ }^{21}$ dependence of the conductivity, and also from the tunneling conductance. ${ }^{22}$

We would like to thank P. W. Anderson, E. I. Blount, D. S. Fisher, and P. A. Lee for helpful discussions and $H$. Dail for help with data acquisition.

(a)Also at Joseph Henry Laboratory, Princeton University, Princeton, N. J. 08544.

${ }^{1}$ P. W. Anderson, Phys. Rev. 109, 1492 (1958).

${ }^{2}$ N. F. Mott, Metal-Insulator Transitions (Taylor and Francis, London, 1974).

${ }^{3}$ J. Hubbard, Proc. Roy. Soc. London, Ser. A 277, 237 (1964), and 281, 401 (1964).

${ }^{4}$ P. P. Edwards and M. J. Sienko, Phys. Rev. 17, 2575 (1978).

${ }^{5}$ N. F. Mott, Philos. Mag. 26, 1015 (1972).

${ }^{6}$ N. F. Mott, Philos. Mag. 44B, 265 (1981).

${ }^{7}$ E. Abrahams, P. W. Anderson, D. C. Licciardello, and T. V. Ramakrishnan, Phys. Rev. Lett. 42,673 (1979), and references therein.

${ }^{8}$ T. F. Rosenbaum, K. Andres, G. A. Thomas, and R. N. Bhatt, Phys. Rev. Lett. 45,1723 (1980); G. A. Thomas, T. F. Rosenbaum, and R. N. Bhatt, Phys。 Rev. Lett. 46, 1435 (1981).

${ }^{9}$ B. W. Dodson, W. L. McMillan, J. M. Mochel, and R. C. Dynes, Phys. Rev. Lett. 46, 46 (1981).

${ }^{10}$ M. Cuevas and H. Fritzsche, Phys. Rev. 137, A1847
(1965), and 139, A1628 (1965). Tuning of $n_{c}$ can also be done with magnetic field as evidenced by the relatively high- $T$ data of R. J. Sladek, J. Phys. Chem. Solids 8 , 515 (1959). W. Sasaki and C. Yamanouchi, J. Non-Cryst. Solids 4 , 183 (1970), used annealing of transmutation doped samples6 - fin

${ }^{11}$ R. N. Bhatt, Phys. Rev. B 24, 3630 (1981).

${ }^{12}$ R. N. Bhatt, to be published, calculated the variation of $n_{c}$ for a Mott transition with a strong central-cell potential, using the method of polarized orbitals to construct the pseudopotential for the one-electron bands. To lowest order, the change in $n_{c}$ is independent of direction for stresses normal to (111).

${ }^{13}$ Y. Ootuka, F. Komori, Y. Monden, S. Kobayashi, and W. Sasaki, Solid State Commun. 36, 827 (1980); T. F. Rosenbaum, K. Andres, and G. A. Thomas, Solid State Commun. 35, 663 (1980).

${ }^{14}$ E. S. Kirkpatrick, Rev. Mod. Phys. 45, 574 (1973).

${ }^{15}$ This constant agrees with that deduced by scaling the theoretical low-stress result (Ref. 12) with the relative change of the isolated donor wave function at high and low stresses, within the uncertainty in sample orientation.

${ }^{16}$ G. A. Thomas, Y. Ootuka, S. Kobayashi, and W. Sasaki, Phys. Rev. B $\underline{24}, 4886$ (1981).

${ }^{17} \mathrm{~A}$. F. Ioffe and A. R. Regel, Prog. Semicon. 4, 237 (1960).

${ }^{18} \mathrm{C}$. Wegner, Z. Phys. B 25,327 (1976); W. L. McMillan, Phys. Rev. B 24, 2739 (1981); D. Vollhardt and P. Wölfle, Phys. Rev. Lett. 45,842 (1980); A. McKinnon and B. Kramer, Phys. Rev. Lett. 47, 1546 (1981); Y. Imry, Phys. Rev。Lett. 44, 469 (1980); D. Belitz, A. Gold, and N. Götze, Z. Phys. B 44, 273 (1981).

${ }^{19}$ T. F. Rosenbaum, K. Andres, G. A. Thomas, and P. A. Lee, Phys. Rev. Lett. $\underline{46}, 568$ (1981).

${ }^{20}$ T. Chui, P. Lindenfeld, W。L. McLean, and K. Mui, Phys. Rev。Lett. 47, 1617 (1981); T. F. Rosenbaum, R. F. Milligan, G. A. Thomas, P. A. Lee, T. V. Ramakrishnan, R. N. Bhatt, K. DeConde, H. Hess, and T. Perry, Phys. Rev. Lett. 47, 1758 (1981).

${ }^{21}$ G. A. Thomas, Y. Ootuka, S. Katsumoto, S. Kobayashi, and W. Sasaki, to be published.

${ }^{22}$ R. C. Dynes and J. P. Garno, Phys. Rev. Lett. 46 , 137 (1981); W. L. McMillan and J. Mochel, Phys. Rev. Lett. 46, 556 (1981); Y. Imry and Z. Ovadyahu, to be published. 